\title{
Caracterización de la satisfacción laboral por género en el personal académico de una Institución de Educación Superior
}

\section{Characterization of job satisfaction by gender in the academic staff of a Higher Education Institution}

\author{
OCHOA-JAIME, Blanca Rosa $\dagger^{*} \&$ VALDEZ-PINEDA, Dina Ivonne \\ Instituto Tecnológico de Sonora. Dirección de Ciencias Económico Administrativa, México.
}

ID $1^{\text {er }}$ Autor: Blanca Rosa, Ochoa-Jaime / ORC ID: 0000-0002-3387-632X, Researcher ID Thomson: S-6886-2018, CVU CONACYT ID: 688073

ID $1^{\text {er }}$ Coautor: Dina Ivonne, Valdez-Pineda / ORC ID: 0000-0001 8660-1787, Researcher ID Thomson: S6886-2018, CVU CONACYT ID: 282771

\begin{abstract}
Resumen
Las Instituciones de Educación Superior deben contribuir en la creación de modelos culturales equitativos, justos y mejores lo cual contribuirá al desarrollo de una sociedad igualitaria de hombres y mujeres. Ante esta prioridad la institución de educación superior del sur de Sonora, México, realizo estudios para conocer las relaciones de equidad de género del personal docente y generar estrategias de intervención para garantizar un espacio democrático e incluyente, apegado a los derechos humanos para todas las personas. Se retoman los resultados obtenidos del proyecto Diagnóstico de Género enfocándose en el personal académico por lo cual se plantea la pregunta ¿Cuál es la relación de equidad de género del personal académico en relación a la satisfacción con el desarrollo personal, reconocimiento laboral, Discriminación, Exclusión/Inclusión, Justicia de procesos y Equidad de la Institución educativa?, siendo su objetivo realizar una caracterización por género del personal académico para conocer las relaciones de equidad y generar estrategias de intervención para garantizar un espacio democrático e incluyente, apegado a los derechos humanos para todas las personas. Se concluye que existen diferencias por género en aspectos como igualdad de oportunidades a puestos jerárquicos, casos de exclusión y discriminación en proyectos y actividades académicas
\end{abstract}

Desarrollo personal, Discriminación, Equidad

\begin{abstract}
Higher Education Institutions must contribute to the creation of equitable, fair and better cultural models, which will contribute to the development of an equal society of men and women. Faced with this priority, the higher education institution in southern Sonora, Mexico, conducted studies to understand the gender equality relations of the teaching staff and generate intervention strategies to guarantee a democratic and inclusive space, attached to human rights for all people. The results obtained from the Gender Diagnosis project are retaken, focusing on academic staff, which is why the question is posed: What is the gender equity relationship of academic staff in relation to satisfaction with personal development, job recognition, Discrimination, Exclusion / Inclusion, Justice of processes and Equity of the educational Institution ?, being its objective to carry out a characterization by gender of the academic staff to know the relations of equity and generate intervention strategies to guarantee a democratic and inclusive space, attached to human rights for everybody. It is concluded that there are differences by gender in aspects such as equal opportunities to hierarchical positions, cases of exclusion and discrimination in projects and academic activities.
\end{abstract}

Personal development, Discrimination, Equity

Citación: OCHOA-JAIME, Blanca Rosa \& VALDEZ-PINEDA, Dina Ivonne. Caracterización de la satisfacción laboral por género en el personal académico de una Institución de Educación Superior. Revista de Gestión Universitaria. 2020. 4-12:2031.

\footnotetext{
* Correspondencia al autor (Correo electrónico: blanca.ochoa@itson.edu.mx)

$\dagger$ Investigador contribuyendo como primer autor.
} 


\section{Introducción}

Las Instituciones de Educación Superior (IES) deben contribuir en la creación de modelos culturales equitativos, justos y mejores para todas las personas; por lo que es primordial la inclusión de una perspectiva de género ya que en el mundo globalizado se han generado ya políticas y recomendaciones desde la década de los ochenta sobre la necesidad de incluir la equidad de género en la formación de nuevos perfiles profesionales, lo cual contribuirá al desarrollo de una sociedad igualitaria de hombres y mujeres, a través de prácticas e implementación de políticas públicas que permita el avance en la sociedad actual (Ochoa, 2011).

Es una prioridad de los organismos internacionales, y de los gobiernos, corregir las desigualdades históricas en las que han estado sumergidas las mujeres, ya que el desarrollo social y el bienestar humano sólo podrán alcanzarse si se incorpora a toda la población y no sólo a la mitad, es decir, si en los procesos de construcción de sociedades sustentables, más justas e igualitarias se incluye el trabajo y el aporte de los hombres y de las mujeres. Esto pone de manifiesto que la igualdad entre mujeres y hombres no es sólo un asunto de justicia social, aunque por sí mismo sería suficiente, sino una necesidad para el desarrollo económico y social de cualquier sociedad (Buquet, 2011; ONU, 2013).

De acuerdo con De Garay y Del Valle (2012), en 14 universidades mexicanas "los hombres tienen un claro dominio en los altos puestos de dirección académica, con el 74\%, lo que pone en evidencia la existencia, aún, del techo de cristal en ese ámbito laboral". Por otro lado, las mujeres académicas continúan dedicando mayor tiempo a actividades del hogar que a actividades laborales, siendo el principal motivo de la toma de decisiones en el ámbito profesional/laboral (Ordorika, 2015).

Ante esta panorámica, la problemática central de una institución de educación superior del sur de Sonora, México, consiste en la necesidad de realizar estudios para conocer las relaciones de equidad de género del personal docente y generar estrategias de intervención para garantizar un espacio democrático e incluyente, apegado a los derechos humanos para todas las personas.

\section{Planteamiento del Problema}

Para poder comprender qué es lo que se sucede en las instituciones, es necesario conocer lo que sucede hacia su interior. La cultura institucional que se refleja a partir de usos, espaciales, lingüísticos y muchos otros, donde se establece lo que es aceptable, adquiridos a través del tiempo y repetición. Estos usos se reproducen sin pensar, tanto al interior, como al exterior.

Para la realización de este trabajo se retoman parte de los resultados obtenidos del proyecto Diagnóstico de Género y para este trabajo se enfocará en los resultados obtenidos del diagnóstico del personal académico por lo cual se plantea la siguiente pregunta de investigación: ¿Cuál es la relación de equidad de género del personal académico en relación a la satisfacción con el desarrollo personal, reconocimiento laboral, Discriminación, Exclusión/Inclusión, Justicia de procesos y Equidad de la Institución educativa?, siendo el objetivo realizar una caracterización por género del personal académico para conocer las relaciones de equidad y generar estrategias de intervención para garantizar un espacio democrático e incluyente, apegado a los derechos humanos para todas las personas.

\section{Desarrollo}

El género, como categoría social surgió para explicar las desigualdades entre hombres y mujeres, poniendo el énfasis en la noción de multiplicidad de identidades ya que lo femenino y lo masculino se conforman a partir de una relación mutua, cultural e histórica. El género es una categoría transdisciplinaria, que desarrolla un enfoque globalizador y remite a los rasgos y funciones psicológicas y socioculturales que se le atribuye a cada uno de los sexos en cada momento histórico y en cada sociedad (Gamba 2008).

Money (1955) propuso el término "papel de género" para describir el conjunto de conductas atribuidas a los varones y a las mujeres, por otro lado (De Barbieri, 1990) define los sistemas de género como los conjuntos de prácticas, símbolos, representaciones, normas y valores sociales que las sociedades elaboran a partir de la diferencia sexual anatómicofisiológica y que dan sentido a las relaciones entre personas sexuadas. 
En general la categoría de género es una definición de carácter histórico y social acerca de los roles, identidades y valores que son atribuidos a varones y mujeres mediante los procesos de socialización, lo cual implica una "relación de poder y asimétrica ya que en general éstas se configuran como relaciones de dominación masculina y subordinación femenina; no se refiere solamente a las relaciones entre los sexos, sino que alude también a otros procesos que se dan en una sociedad: instituciones, símbolos, identidades, sistemas económicos y políticos, etc. y además es transversal ya que se articulan con otros factores como la edad, estado civil, educación, etnia, clase social, etc.".

La perspectiva de género se puede definir como la introducción de una mirada particular en la comprensión de la vida social, a partir de la idea de que ser hombre o ser mujer es un dato cultural, no meramente biológico, y que la forma que adoptan las desigualdades sociales basadas en el sexo de las personas se relaciona con la manera como se construye la oposición hombre/mujer en el imaginario social, por lo que afirma, los conceptos de género determinados desde una perspectiva biológica, se han naturalizado en la sociedad, que forman parte de nuestras estructuras cognitivas y de todo proceso de significación de forma prácticamente inadvertida, por lo que operan de manera velada, garantizándose, por esto mismo, su eficacia. Equidad de género en este contexto implica que tanto mujeres como hombres, independientemente de sus diferencias biológicas, tienen derecho a acceder con justicia e igualdad al uso, control y beneficio de los mismos bienes y servicios de la sociedad, así como a la toma de decisiones en los ámbitos de la vida social, económica, política, cultural y familiar.

Es la aceptación de las diferencias entre hombres y mujeres y la aceptación de los derechos, buscando el ideal de un equilibrio en el que ninguno de ambos sexos se beneficie de manera injusta en perjuicio del otro (Ochoa, 2011). Por ende, la propuesta de la equidad entre los sexos, es decir, la equidad en las relaciones de género implica la participación equivalente de varones y mujeres en el trabajo, la educación, la vida política y la familia (Miranda, 2007).
De acuerdo con la Ley General para la Igualdad entre Mujeres y Hombres, la perspectiva de género se refiere a la metodología y los mecanismos que permiten identificar, cuestionar y valorar la discriminación, desigualdad y exclusión de las mujeres, que se pretende justificar con base en las diferencias biológicas entre mujeres y hombres, así como las acciones que deben emprenderse para actuar sobre los factores de género y crear las condiciones de cambio que permitan avanzar en la construcción de la igualdad de género.

Esta perspectiva ayuda a comprender más profundamente tanto la vida de las mujeres como la de los hombres y las relaciones que se dan entre ambos. Este enfoque cuestiona los estereotipos con que somos educados y abre la posibilidad de elaborar nuevos contenidos de socialización y relación entre los seres humanos. El empleo de esta perspectiva plantea la necesidad de solucionar los desequilibrios que existen entre mujeres y hombres, mediante acciones como una redistribución equitativa de las actividades entre los sexos (en las esferas de lo público y privado), una Justa valoración de los distintos trabajos que realizan mujeres y hombres, especialmente en lo referente a la crianza de las hijas e hijos, el cuidado de los enfermos y las tareas domésticas lo cual debe implicar una modificación de las estructuras sociales, los mecanismos, las reglas, prácticas y valores que reproducen la desigualdad así como el fortalecimiento del poder de gestión y decisión de las mujeres.

\section{Metodología}

La Investigación de este estudio fue de corte cuantitativa y transversal, donde la población objetivo de este diagnóstico es el personal académico tanto del género femenino como del masculino de una Institución de Educación Superior del Sur de Sonora, México. La población académica se constituye de 1,463 docentes que incluyen Profesores/as de Tiempo Completo indeterminados (PTC), y determinados (eventuales), así como docentes auxiliares y técnicos/as académicos/as. La muestra1 se constituyó por 554 docentes, lo cual representa el $37.9 \%$ de la población total de docentes de las Direcciones. 


\section{Modelo métrico para la equidad de género}

El instrumento de medida para el personal académico consiste en una adaptación del propuesto por Buquet, Cooper y Rodríguez (2010) para medir equidad de género en educación superior. Dicho instrumento cuenta con diecinueve reactivos de atributos personales, familiares y escolares, que incluye áreas de conocimiento, los niveles educativos en que se ejerce la docencia, la participación en espacios de decisión, y la obtención de premios y estímulos.

Después se tiene la escala para medir las diferencias en desarrollo profesional con once reactivos para personal académico. Enseguida se ubica una escala de seis reactivos que mide la dimensión estímulos y reconocimiento al trabajo académico. Se incluyen tres preguntas sobre percepción de discriminación por género. Otros nueve reactivos miden la probabilidad de comentarios públicos de discriminación por género en académicos.

Contiene dos reactivos sobre obstáculos para el desarrollo personal. Finalmente tenemos una escala de veintiún reactivos que miden exclusión inclusión, justicia en los procesos y equidad de género. Esta última escala es original de Maranto y Griffin (2011). El total de reactivos para personal académico fue 71 .

\section{Análisis factoriales de las escalas de medida}

Los resultados del análisis factorial exploratorio fueron llevados a cabo a través de una extracción de máxima verosimilitud y una rotación Oblimin directa. El criterio de aceptación para cada uno de los reactivos fue de un peso factorial mayor o igual a 0.35 , los valores de la prueba de KáiserMeyer-Olkin (KMO) fueron satisfactorias para todas y cada una de las escalas que fueron analizadas y de las dimensiones obtenidas.

El análisis factorial incluyó todos los reactivos de la escala de medida, como se apunta en la tabla 1, la satisfacción con el desarrollo profesional generó en la gráfica de saturación dos dimensiones, la de mayor número de reactivos se refiere a aquellos de tipo personal como el respeto, el ambiente, el reconocimiento, el espacio, la capacitación, y después viene una segunda dimensión que es la de la institución.
Para docentes se observan estas dos dimensiones con diferentes números de reactivos, ya que el reactivo adicional que pertenece al desarrollo personal que se aplicó a los/as docentes fue el nivel de apoyo para la actividad docente/investigación.

Los reactivos de reconocimiento laboral y la escala de discriminación son unidimensionales la frecuencia de comentarios públicos discriminatorios aun cuando es unidimensional para personal docente.

La escala de exclusión-inclusión, la cual tiene cuatro reactivos de inclusión y tres reactivos de exclusión laboral. La escala de justicia de procesos es unidimensional. Finalmente, la escala de equidad tiene nueve reactivos para docentes y dos dimensiones, una de equidad que tiene seis reactivos, y la de inequidad, que tiene tres reactivos. Tabla 1

\begin{tabular}{|l|r|r|r|r|r|}
\hline \multicolumn{1}{|c}{$\begin{array}{c}\text { Nombre de la } \\
\text { escala }\end{array}$} & \multicolumn{1}{c}{$\begin{array}{c}\text { \# de } \\
\text { Reactivos }\end{array}$} & \multicolumn{1}{c}{ KMO } & \multicolumn{1}{c|}{$\begin{array}{c}\text { Varianza } \\
\text { Explicada }\end{array}$} & \multicolumn{1}{|c|}{$\begin{array}{c}\text { \#de } \\
\text { factores }\end{array}$} & \multicolumn{1}{c|}{$\begin{array}{c}\text { \# de } \\
\text { reactivos }\end{array}$} \\
\hline $\begin{array}{l}\text { Satisfacción con } \\
\text { el desarrollo } \\
\text { personal }\end{array}$ & 11 & 0.887 & 52.28 & 2 & $7 / 4$ \\
\hline $\begin{array}{l}\text { Reconocimiento } \\
\text { laboral }\end{array}$ & 6 & 0.617 & 37.78 & 1 & 6 \\
\hline Discriminación & 3 & 0.663 & 47.1 & 1 & 3 \\
\hline $\begin{array}{l}\text { Exclusión- } \\
\text { inclusión }\end{array}$ & 7 & 0.700 & 34.2 & 2 & $4 / 3$ \\
\hline $\begin{array}{l}\text { Justicia de } \\
\text { procesos }\end{array}$ & 5 & 0.879 & 5.49 & 1 & 5 \\
\hline Equidad & 9 & 0.849 & 38.7 & 2 & $6 / 3$ \\
\hline
\end{tabular}

Tabla 1 Resultados del análisis factorial para las escalas de la Medida Equidad de Género del ITSON

Fuente: Elaboración propia a partir de los resultados

\section{Procedimiento}

Una vez obtenido el tamaño de muestra se ubicó un espacio cómodo y confortable y bien iluminado para la aplicación de los cuestionarios para el personal docente. El departamento de personal fue el encargado de citar a los/as empleados/as que eran elegidos/as de manera aleatoria y a cada uno/a se les indicaba la hora y día específico para el llenado de su cuestionario, Para la muestra se utilizaron las salas de lectura con una capacidad de ciento veinte personas y fueron divididas en tres segmentos de cuarenta personas cada una, y colocadas las mesas de tal manera que los/as participantes pudieran estar lo más lejos posible para evitar interrupciones, pláticas o conversaciones vinculadas con la respuesta al cuestionario. 
Los cuestionarios fueron aplicados por medio de dos investigadores/as capacitados/as y entrenados/as para la aplicación y solución de dudas acerca del cuestionario. En todos los casos, los académicos/as respondieron la encuesta en un solo momento, de manera continua y no fue posible que la llevaran a casa. A cada uno de ellos/as se le entregó el cuadernillo y se le indicó la manera en la cual se deberían de llenar los datos de identificación, después de la cual, procedieron a llenar el instrumento de medida.

El equipo total de entrevistadores/as y capacitadores/as fue de ocho asistentes y cuatro para monitorear. Todos ellos/as debidamente estandarizados/as para evitar errores y llevar a cabo la aplicación de manera estandarizada.

Todos/as los/as participantes firmaron un consentimiento informado que les indicaba los objetivos, participación voluntaria y con quién dirigirse para resolver dudas y sugerencias.

\section{Resultados}

A continuación, se describen los resultados de los contrastes de hipótesis que se generan toda vez que cada una de las dimensiones resultantes de la sumatoria de los reactivos involucrados se divide entre el número de reactivos y se establece una variable continua que nos permite llevar a cabo contrastes de hipótesis paramétricas para cada uno de los grupos diferenciados por sexo, edad y otras variables de atributo de cada uno de los grupos.

\section{Diferencias significativas de los atributos del personal académico}

En relación con el personal académico, se encontró que el $14.6 \%$ de las mujeres pertenecen al sindicato comparado con un $29 \%$ de los hombres $\left(\chi^{2}, \mathrm{p}=.000\right)$; también la antigüedad como auxiliar fue mayor para mujeres $(31.5 \%)$ comparado con los hombres $(21.9 \%)$ con una significancia estadística de $\mathrm{p}=.005$.
Siguiendo con los estímulos, encontramos que el $27.3 \%$ de las mujeres perciben estímulos al desempeño, comparado con el $36.3 \%$ de los hombres $(\mathrm{p}=.01)$; son acreedoras/es al perfil PRODEP el 17.9\% de las mujeres y el $28.6 \%$ de los hombres $(\mathrm{p}=.002)$; finalmente colaboran con otros/as investigadores/as el $34.4 \%$ de las mujeres y el $44.1 \%$ de los hombres ( $\mathrm{p}=.007)$. En términos de nombramiento académico sólo el $14.9 \%$ de las mujeres es docente investigador, contrastado con un $25.7 \%$ de los hombres, mientras que el $52.3 \%$ de las mujeres es docente auxiliar, contrastado con el 49.4\% (p=.02) de los hombres. En la categoría de titular encontramos al $17 \%$ de las mujeres y al $29 \%$ de los hombres, en la categoría de auxiliar al 60\% de las mujeres y al 57\% de los hombres $(\mathrm{p}=.000)$.

Adicionalmente, tenemos que el $17 \%$ de las mujeres tiene un contrato indeterminado comparado con el 30\% de los hombres, mientras que el $70.6 \%$ de las mujeres tiene un contrato por tiempo determinado comparado con un $55 \%$ de los hombres. Existe una diferencia significativa en el estado civil ya que el porcentaje de divorciados hombres es el 5\% mientras que el $10 \%$ es de divorciadas mujeres, el $22 \%$ está soltero comparado con $30 \%$ de las mujeres, mientras que el $66 \%$ de los hombres está casado, comparado con el 55\% de las mujeres.

En resumen, en la parte atributiva podemos observar que existen diferencias significativas importantes en principio en el tipo de contratación, en el nivel en el que se encuentran hombres y mujeres, en la categoría, en el tipo de nombramiento académico y en la pertenencia al sindicato, en relación a los estímulos en docentes encontramos también proporciones estadísticamente significativas que hacen ver que la mujer tiene menos acceso a los estímulos al desempeño, a las horas extras y a los pagos adicionales.

\section{Contraste de hipótesis para el personal académico}

Las pruebas de contraste de hipótesis para factores dicotómicos y las variables continúas relacionadas con las dimensiones de las escalas que se aplicaron en el cuestionario, fueron llevadas a cabo a través de pruebas $t$ de student adicionando la prueba de Levene para asumir varianza iguales o desiguales. 
Es necesario indicar que para cada uno de los factores que vamos a mencionar se llevaron a cabo los contrastes de hipótesis para todas y cada una de las variables consideradas, que son trece, y solamente mencionaremos aquellas que hayan resultado con diferencias significativas en sus medias para cada uno de los factores analizados.

Para la variable sexo resultaron con diferencias significativas los estímulos y reconocimientos al trabajo académico con una significancia bilateral de $\mathrm{p}=.007$ y con una ventaja para los hombres. Se encontraron también diferencias significativas en la dimensión discriminación, que está constituida por tres reactivos, con una percepción mayor para las mujeres $(1.81$; D.S. $=1.0)$, finalmente perciben procesos más injustos las mujeres comparadas con los hombres con una significancia bilateral de $\mathrm{p}=.003$.

Los resultados del análisis de varianza de una sola vía con la prueba post hoc de Scheffé, en donde para cada uno de los factores siguientes: grado académico, categoría, tipo de contrato, nombramiento académico y nivel; en todos ellos resultaron con un nivel estadísticamente significativo particularmente la satisfacción con el desarrollo personal y la dimensión de equidad. Las demás dimensiones no resultaron significativas y por tanto no se mencionan.

En relación con los resultados de la prueba post hoc de Scheffé, se encontró acerca del grado académico, quienes perciben mayor apoyo institucional son las personas que tienen doctorado, y quienes tienen menor percepción de satisfacción con su desarrollo personal son los que tiene estudios de maestría.

En la percepción de equidad laboral encontramos que quienes perciben mayor equidad son aquellos/as que tienen licenciatura, y quienes perciben menor equidad son aquellas personas que tienen doctorado. En categoría, encontramos que quienes perciben mayor satisfacción con el desarrollo personal son los/as maestros/as investigadores/as de tiempo completo, mientras que los/as que perciben menor desarrollo son los/as técnicos/as académicos/as.
En relación con la equidad laboral, los que perciben menor equidad son los/as técnicos/as académicos/as y los que perciben mayor equidad son los y las docentes auxiliares.

En relación con el tipo de nombramiento, son los/as docentes titulares los/as que perciben mayor satisfacción con su desarrollo y también mayor equidad laboral, mientras que los/as auxiliares son los/as que perciben menor satisfacción y equidad. En relación con el tipo de contrato, los/as docentes indeterminados/as perciben mayor satisfacción y equidad laboral, y los/as eventuales menor satisfacción y equidad laboral.

En relación con el nivel en que se encuentran, los y las docentes titulares $\mathrm{C}$ son quienes perciben mayor desarrollo personal y mayor equidad laboral, mientras que los y las docentes auxiliares perciben menor equidad y satisfacción con el desarrollo personal.

Es importante que estas variables sean las que resultan significativas después de la comparación por sexo que se llevó a cabo a través del análisis $t$ de student. Por otro lado, los factores dicotómicos: contar con el perfil PRODEP, estar inscrito al Sistema Nacional de Investigadores (SNI) recibir compensación por algún puesto administrativo o en su caso colaborar con colegas extranjeros/as para llevar a cabo actividades académicas $o$ de investigación, no generan ningún tipo de diferencia dentro de las variables asociadas a discriminación, exclusión, inclusión, equidad o justicia de procesos.

\section{Análisis descriptivos para el personal académico comparando las diferentes direcciones}

Para realizar el análisis descriptivo se obtienen los estadísticos descriptivos para hombres y después para mujeres, se contrastan a través de una prueba $t$ de student para evaluar la significancia de la diferencia entre medias de hombres y mujeres, y después de esto se lleva a cabo un análisis de varianza simple de una sola vía y efectos aleatorios para conocer si existen diferencias dentro del grupo de hombres y el grupo de mujeres en relación a las seis Direcciones con las que cuenta la institución. 
En la dimensión que hemos denominado reconocimiento laboral que incorpora preguntas sobre el esfuerzo, el reconocimiento, el trabajo grupal y gremial en relación con los obstáculos y los esfuerzos que representa para hombres y mujeres, encontramos que en todas las Direcciones se percibe una diferenciación, aunque no estadísticamente significativa para las mujeres en comparación con los hombres.

Particularmente en el área de Cs. Sociales que corresponde a la media más alta de los hombres, se observa una media de 3.13 para hombres y la media más alta para las mujeres es en la Dirección Navojoa con 3.07.

Estos valores de media que giran alrededor de tres indican una percepción que pasa de un moderado acuerdo a un moderado desacuerdo, y que en la medida en que incrementa su cantidad aumenta el nivel de acuerdo con las aseveraciones del cuestionario; ese aumento está relacionado con un aumento en el nivel de esfuerzo, en las ocupaciones o en el trabajo dedicado por parte de hombres y mujeres.

En la dimensión que hemos denominado discriminación y que incluye tres reactivos que se centran en la discriminación dentro de la Institución, ya sea por comisiones evaluadoras o por otorgar permisos o promociones, encontramos que el valor máximo de media es de dos en la Dirección de Cs. Sociales para mujeres, el valor menor de 1.5 lo encontramos en la Dirección de Ingeniería y Tecnología, también para el caso de las mujeres.

Los resultados con valores de media que oscilan entre $1.5 \mathrm{y}$ dos nos indican que hay una baja frecuencia de discriminación, pero desde la perspectiva de los docentes, particularmente de las mujeres, asumen que tal discriminación está cercana a dos.

En general sería una labor de la Institución disminuir estos valores de percepción de discriminación que son estadísticamente diferentes con un nivel de $\mathrm{p}=.001 \mathrm{y}$ un valor de $\mathrm{t}=3.45$ entre hombres y mujeres.
En la dimensión de inclusión laboral en donde se encuentran reactivos como las oportunidades para trabajar en la investigación, la satisfacción con la vida personal, sentirse incluido en las reuniones sociales informales, etc., encontramos que son las mujeres de la Dirección de Ingeniería y Tecnología las que exhiben la media más alta con 4.41 indicando un completo acuerdo con su perspectiva de inclusión laboral. Por otro lado, el valor más bajo de 3.70 corresponde a los hombres de Cs. Económico-Administrativas y aun cuando no existen diferencias significativas entre hombres y mujeres a través de las diferentes Direcciones, encontramos siempre valores menores para las mujeres excepto en la Dirección de Ingeniería y Tecnología. El contraste de hipótesis para hombres y mujeres en esta dimensión no resultó significativo.

En lo referente a la exclusión laboral que incluye a aquellos reactivos como: me siento aislado/a, los/as colegas no comparten conmigo la información que comparten con los demás, y otros, encontramos que el valor más alto de exclusión que implica un moderado desacuerdo se ubica entre los hombres de Ingeniería y Tecnología con un valor de media de 2.67.

En esta dimensión de exclusión laboral se supone que los valores más bajos se relacionan con un desacuerdo a que existe exclusión laboral, de tal forma la diferencia que encontramos en Recursos Naturales para hombres con una media de 1.82 y de 2.54 para las mujeres, resulta en una brecha bastante amplia en donde las mujeres asumen que existe un buen nivel de exclusión laboral ya que se acercan mucho al valor de tres, lo mismo para las mujeres de Ingeniería y Tecnología, inclusive para los hombres también de esa Dirección.

En relación con la igualdad de oportunidades, la asignación de horas, cursos, etc., a lo que llamamos la dimensión de justicia de procesos encontramos que existe un moderado acuerdo en la mayoría de los/as docentes de las distintas Direcciones. Particularmente la Dirección de Cs. Sociales es en donde se encuentra la brecha más amplia entre hombres y mujeres, con una media de 3.92 para los hombres y 3.20 para las mujeres. 
Es en esta dimensión en donde encontramos un valor de $\mathrm{p}=.05 \mathrm{y} \mathrm{t}=1.96$ para la comparación de hombres y mujeres, lo cual nos indica la urgencia de trabajar con las brechas entre hombres y mujeres y aumentar los valores por arriba de cuatro para asumir que existe justicia de procesos dentro de la Institución.

La dimensión de equidad laboral se encuentra en niveles bastante satisfactorios por arriba de cuatro y hasta 4.5. El valor más bajo lo encontramos en Cs. Sociales en las mujeres con una media de 3.78, es en esta Dirección en donde encontramos la brecha más amplia de 4.27 de media para hombres y 3.78 para mujeres.

Para la última dimensión inequidad laboral, donde los valores cercanos a uno son los óptimos, valores que van del 1.5 al 1.95 y que nos indican que hay un moderado desacuerdo en relación a la inequidad laboral. Para la mayoría de las Direcciones el valor aumenta para las mujeres, excepto en Ingeniería y Tecnología, sin embargo, las pruebas de hipótesis no mostraron que existieran diferencias estadísticamente significativas entre hombres y mujeres en las diferentes Direcciones.

\section{Indicadores de equidad de género}

En el presente apartado se describe la situación del personal académico a través de diferentes indicadores de entrada, tanto cuantitativos como cualitativos, con el objetivo de observar la presencia de equidad o inequidad entre hombres y mujeres pertenecientes a estas poblaciones, así como identificar en dónde ocurren estas diferencias.

El análisis de la información obtenida por medio de la aplicación de la Medida de Equidad de Género en Educación Superior y de los registros proporcionados por la Institución se realiza por dimensiones, las cuales agrupan distintos tipos de indicadores que refieren a situaciones específicas; siguiendo la estructura propuesta por Buquet, Coooper y Rodríguez (2010). La estructura de los indicadores.
Indicadores de entrada cuantitativos para el personal académico

El personal académico está integrado por profesores/as de tiempo completo (PTC), con contrato indeterminado y determinado; así como por docentes auxiliares y técnicos/as académicos/as pertenecientes a las diferentes Direcciones académicas del ITSON. Los primeros dos indicadores que se presentan se obtienen a partir de la población total, y el resto de los indicadores se derivan de la muestra que participó en el proyecto de Diagnóstico de Equidad de Género, la cual se constituye por 554 académicos/as, 309 mujeres y 245 hombres.

\section{Igualdad de oportunidades en el acceso al empleo académico}

El personal académico está compuesto por 1,463 docentes, cuya distribución entre hombres y mujeres es muy similar, sólo existe una diferencia de $1.2 \%$ (17 personas) a favor de los hombres, por lo que la población académica es mixta y refleja de forma global oportunidades laborales para ambos sexos (ver tabla 2).

\begin{tabular}{|l|r|r|}
\hline \multicolumn{1}{|c|}{ Sexo } & Cantidad & Porcentaje \\
\hline Mujeres & 723 & $49.40 \%$ \\
\hline Hombres & 740 & $50.60 \%$ \\
\hline Total & 1463 & $100 \%$ \\
\hline
\end{tabular}

Tabla 2 Porcentaje de mujeres y de hombres en el total de personal académico

Fuente: Elaboración propia con base en los registros del personal académico

Se calculó el índice de contribución al sexismo (ISC) para observar la participación de hombres y mujeres al interior de la institución de educación superior (IES), en cada una de sus Direcciones y el grado en que cada una de ellas contribuye a reproducirlo. Cuando el índice es positivo favorece a la masculinización y cuando es negativo favorece a la feminización, y la contribución es mayor cuando el valor es superior a uno.

La Dirección de Ingeniería y Tecnología es la que genera una brecha más grande entre hombres y mujeres con un ISC de 7, a favor de la masculinización, se advierte también que la cantidad de docentes hombres que laboran en esta Dirección dobla a la cantidad de mujeres. 
Por su parte las Direcciones de Cs. Económico-Administrativas y Sociales cuentan con un ISC negativo mayor a uno, particularmente la primera de ellas con -3.76, lo cual significa que la distribución de docentes hombres y mujeres en estas Direcciones impacta sobre la feminización.

\section{Participación en redes profesionales}

De acuerdo con los resultados de la encuesta en relación a la colaboración de los/as docentes con colegas para el desarrollo de sus actividades académicas y de investigación, los hombres reportan tener un mayor vínculo con otros/as profesionales, cerca del 50\% de los encuestados, mientras que una tercera parte de las mujeres participantes colabora con colegas de otras instituciones en su quehacer académico.

\section{Exclusión por motivos de sexo}

En cuanto a la proporción docentes que informaron haber sido excluidos/as en alguna ocasión de una actividad (premio, permiso, promoción, entre otras) por ser hombre o ser mujer, la proporción de mujeres que considera haber vivido exclusión por motivos de sexo está cercana a la tercera parte de la muestra consultada (31.1\%); la proporción de los hombres que se han sentido excluidos por la misma razón se encuentra por debajo de la quinta parte $(18.8 \%)$. Se observa pues que desde la perspectiva de los/as consultados/as existe una mayor exclusión hacia el personal docente femenino en cuanto a la participación en los diferentes eventos académicos (ver tabla 3 ).

\begin{tabular}{|l|r|r|}
\hline \multicolumn{1}{|c|}{ Sexo } & Cantidad & Porcentaje \\
\hline Mujeres & 96 & $31.1 \%$ \\
\hline Hombres & 46 & $18.8 \%$ \\
\hline
\end{tabular}

Tabla 3 Proporción de mujeres y de hombres que han sido excluidas/os de algún evento académico

Fuente: Elaboración propia con base en los resultados de la medida de Equidad de Género

\section{Indicadores de entrada cualitativos para el personal académico}

Los indicadores a los que se hace mención en este apartado son los que se pueden obtener a través del instrumento de medida aplicado, aun cuando son cualitativos tienen una expresión numérica y se refieren a percepciones $u$ opiniones sobre determinadas situaciones que se dan al interior de la IES.
Igualdad de oportunidades en el acceso al empleo académico

\section{Disponibilidad de recursos}

En la tabla 4 se presenta la proporción de docentes que consideran que los recursos con los que cuentan para realizar sus actividades son adecuados. La proporción de mujeres que percibe de suficientes a excelentes los recursos (materiales y tecnológicos) que tiene disponibles es ligeramente mayor al de los hombres; en general tres cuartas partes de los participantes, poco más para las mujeres, poco menos para los hombres, manifiesta un nivel alto de aceptación de los recursos de que dispone. Sería importante conocer por qué el $23.3 \%$ de las mujeres y el $26.9 \%$ de los hombres encuestados/as no considera sus recursos (equipo, espacio, etc.) como suficientes, una circunstancia relevante para el desarrollo de las actividades académicas y el empoderamiento.

\begin{tabular}{|l|r|r|}
\hline \multicolumn{1}{|c|}{ Sexo } & Cantidad & Porcentaje \\
\hline Mujeres & 237 & $76.6 \%$ \\
\hline Hombres & 179 & $73.1 \%$ \\
\hline
\end{tabular}

Tabla 4 Proporción de mujeres y de hombres que perciben que sus recursos son adecuados para el desarrollo de sus labores académicas

Fuente: Elaboración propia con base en los resultados de la medida de Equidad de Género

\section{Percepción de evaluaciones o dictámenes}

La proporción de docentes mujeres y hombres que han sentido en alguna ocasión haber recibido una evaluación o dictamen injusto por parte de comisiones dictaminadoras o evaluadoras se presenta en la tabla 5; tanto mujeres como hombres se acercan al $40 \%$ de los/as participantes en el estudio con una percepción de que su trabajo académico ha recibido una evaluación parcial, una proporción alta.

\begin{tabular}{|l|r|r|}
\hline \multicolumn{1}{|c|}{ Sexo } & Cantidad & Porcentaje \\
\hline Mujeres & 118 & $38.2 \%$ \\
\hline Hombres & 92 & $37.6 \%$ \\
\hline
\end{tabular}

Tabla 5 Proporción de mujeres y de hombres que perciben que la evaluación o dictamen sobre su trabajo académico ha sido injusta

Fuente: Elaboración propia con base en los resultados de la medida de Equidad de Género 


\section{Discriminación en el ámbito laboral}

En cuanto al indicador de discriminación, se destaca que poco más de la mitad de las docentes mujeres encuestadas percibe que existe discriminación por razones de género en el lugar donde laboran, en el caso de los hombres la proporción disminuye al $38.4 \%$. Estas altas proporciones dan cuenta de una percepción de trato desigual o menos favorable con base en el sexo de las personas y que las sitúa en una posición de desventaja, particularmente a las mujeres como se observa en la tabla 6 , lo que puede resultar en limitaciones en el acceso a oportunidades, reconocimiento, recursos, etc.

\begin{tabular}{|l|r|r|}
\hline \multicolumn{1}{|c|}{ Sexo } & Cantidad & Porcentaje \\
\hline Mujeres & 156 & $50.5 \%$ \\
\hline Hombres & 94 & $38.4 \%$ \\
\hline
\end{tabular}

Tabla 6 Proporción de mujeres y de hombres que perciben discriminación en su Institución

Fuente: Elaboración propia con base en los resultados de la medida de Equidad de Género

\section{Satisfacción en el trabajo}

En relación con el indicador de satisfacción del personal académico en el lugar donde laboran, los hombres muestran una proporción mayor en este rubro con $77.6 \%$, las mujeres no se alejan mucho, casi tres cuartas partes de las entrevistadas se sienten satisfechas en su trabajo (ver tabla 7). Estar satisfecho/a o no en el trabajo puede incidir en la forma de llevar a cabo las obligaciones laborales, el $25.9 \%$ de las mujeres y el $22.4 \%$ de los hombres consultados/as no se sienten satisfechos/as en el trabajo.

\begin{tabular}{|l|r|r|}
\hline \multicolumn{1}{|c|}{ Sexo } & Cantidad & Porcentaje \\
\hline Mujeres & 229 & $74.1 \%$ \\
\hline Hombres & 190 & $77.6 \%$ \\
\hline
\end{tabular}

Tabla 7 Proporción de mujeres y de hombres que se sienten satisfechas/os en el trabajo

Fuente: Elaboración propia con base en los resultados de la medida de Equidad de Género

\section{Conclusiones}

Los resultados obtenidos en base a las pruebas realizadas permiten cumplir con el objetivo establecido para este estudio. Basado en eso, se obtuvieron una serie de conclusiones.
Igualdad de oportunidades en el acceso al empleo

Existe mayor oportunidad para ingresar como personal docente auxiliar tanto para hombres como para mujeres, siempre y cuando cumplan con los requisitos que básicamente son contar con título y cedula profesional, así como experiencia en el campo laboral relacionado con la asignatura que se desea impartir. Esta ha sido un área de oportunidad para profesionistas mujeres principalmente, de diversas disciplinas que por tener uno o más hijos/as pequeños/as consideran como buena opción renunciar en sus trabajos de tiempo completo y dar algunas clases en ITSON en horarios accesibles para poder cumplir con su rol en el hogar, uno de los aspectos a destacar en cuanto al trabajo de las mujeres en la docencia es su desigual participación en relación con los varones en los cargos jerárquicos (Bonder, 1994).

En la institución lo anterior sigue siendo una realidad, ya que a pesar de que gran porcentaje de mujeres laboran como docentes y tienen puestos como Responsables de Programa $\mathrm{u}$ otros mandos medios, su presencia en altos niveles jerárquicos es escasa, por ejemplo las profesoras mencionan que nunca ha habido rectora, y solo reportan haber conocido a una vicerrectora; pero consideran que existe personal académico con formación y experiencia en la Institución para ocupar dichos puestos en el organigrama.

Lo anterior coincide con lo encontrado hace más de una década en el medio académico del Reino Unido por Morley (1999), tener mujeres altamente calificadas y con educación costosa concentradas en los niveles más bajos, constituye un mal manejo de recursos, desperdiciando capacidades. Según refieren las profesoras y algunos profesores la institución invierte en la formación continua de su personal académico tanto de manera interna como externa, eso no garantiza que se recupere el gasto de dicha inversión, ya que abre pocos espacios para contrataciones por tiempo indeterminado y/o plazas académicas, lo cual en ocasiones promueve la llamada "fuga de cerebros", propiciando que profesores o profesoras opten por irse a laborar a otra institución donde pueda tener mayores prestaciones y beneficios. 


\section{Satisfacción en el trabajo y clima laboral}

En cuanto a la percepción de los y las docentes sobre el clima de trabajo se tiene que para la mayoría existe cordialidad, ambientes de trabajo amenos, colaborativos, sin embargo, algunas de las cuestiones por mejorar son la segmentación de grupos de docentes por afinidades, lo cual, aunque no es percibido como algo negativo, consideran que a veces suelen presentarse casos de exclusión en proyectos o actividades de la academia por no ser parte del mismo grupo o por ser docente auxiliar. Una cuestión que debe analizarse en conjunto por el personal docente y directivo es el uso y funciones de los grupos de WhatsApp, debido a que existen problemas de comunicación o inconformidades sobre los tipos de mensajes que se envían y los horarios, así como cuestiones como la privacidad de cada docente.

\section{Toma de decisiones y empoderamiento de las} profesoras

Dentro de la visión y acción de la educación superior para el siglo XXI planteada por la UNESCO (1998) en el artículo 4 se encuentra lo referente al fortalecimiento de la participación y promoción del acceso de las mujeres. Casi veinte años después, aunque se hayan realizado progresos considerables en cuanto a mejorar el acceso de las mujeres a la enseñanza superior, lograr su incursión en licenciaturas o ingenierías estereotipadas como masculinas, sigue siendo una prioridad urgente en el proceso de renovación encaminado a establecer un sistema de educación superior equitativo y no discriminatorio. Asimismo, hay que esforzarse por eliminar los obstáculos políticos y sociales que hacen que la mujer esté insuficientemente representada, y favorecer la participación de la mujer en los niveles de la elaboración de políticas y toma de decisiones, tanto en la universidad como a nivel personal.

El personal docente manifestó en los grupos focales la necesidad de lo anterior, ya que principalmente la participación en toma de decisiones por parte de docentes auxiliares es nula en los procesos de la Institución, solo una minoría ha participado en Diseños curriculares, sin embargo, principalmente las profesoras expresan sentimientos de exclusión en aquellas tomas de decisiones que afectan su área de trabajo en ITSON.

\author{
Empoderamiento y autonomía de las \\ trabajadoras
}

Las docentes expresan necesidades que deben cubrirse para propiciar su empoderamiento y autonomía, como contar con servicio médico para sus conyugues, contar con una guardería dentro del campus o cerca, ya que se les da apoyo económico para guardería, sin embargo, la mayoría de ellas deja sus hijos o hijas al cuidado de familiares, por la falta de espacios en guarderías de la ciudad o la distancia.

\section{Recomendaciones}

Propiciar iniciativas para reducir las diferencias en proporciones de docentes mujeres y hombres en puestos o contratos indeterminados.

Promover desde la alta dirección, la participación de las mujeres en puestos directivos o de alto nivel en la institución, a partir de los méritos y perfil para los puestos sin que exista rivalidad de sexos.

Finalmente es recomendable realizar un análisis de normas y reglamentos de la Institución desde una perspectiva de género y derechos humanos, así como adecuarlos desde dichos enfoques, tanto para garantizar un trato equitativo y justo a estudiantes como a docentes y personal administrativo o de servicios que labora en la institución. Así mismo, se recomienda actualizar instrumentos de evaluación docentes y al personal no docente desde la perspectiva de derechos humanos y equidad de género.

\section{Referencias}

Alfaro, J. (2000). Discusiones en psicología comunitaria. Santiago, Chile: Universidad Diego Portales.

Antolín, L. (2003) La mitad invisible. Género en la educación para el desarrollo. Madrid: ACSUR-Las segovias.

Bonder, G. (1994). Mujer y Educación en América Latina: hacia la igualdad de Oportunidades. Revista Iberoamericana de Educación. Número 6. Recuperado de http://rieoei.org/oeivirt/rie06a01.htm 
Buquet, A. (2011). Transversalización de la perspectiva de género en la educación superior. Problemas conceptuales y prácticos. Perfiles Educativos, vol. XXXIII, pp. 211-225.

Comisión Nacional para Prevenir y Erradicar la Violencia Contra las Mujeres (2018). ¿Qué es la perspectiva de género y por qué es necesario implementarla? Recuperado de https://www.gob.mx/conavim/articulos/que-esla-perspectiva-de-genero-y-por-que-esnecesario-implementarla

De Garay, A. y Del Valle, Gabriel. (2012). Una mirada a la presencia de las mujeres en la educación Superior en México. Revista Iberoamericana de Educación Superior, 3(6), 330 .

INMUJERES (2003). Equidad de género y medio ambiente. Instituto Nacional de las Mujeres. México, D.F.

Maranto, Ch., Griffin, A. (2011). The antecedents of a 'chilly climate' for women faculty in Higher Education. Human Relations, 64(2), pp .139-159. DOI: $10.1177 / 0018726710377932$

Miranda, G. R. (2007). Mujeres, educación superior e igualdad de género CPU-e, Revista de Investigación Educativa, (4), 1-30. Recuperado de

http://www.redalyc.org/pdf/2831/28312171000 4.pdf

Morley, L. (1999). Techo de cristal o jaula de hierro. Las mujeres en el ámbito académico del Reino Unido. En: Belausteguigoitia, M., y Mingo, A (Eds.). Géneros prófugos. Feminismo y educación. Pp. 349-368. México: UNAM, Colegio de la Paz Vizcaínas, PAIDÓS.

Ochoa, C. (2011). Género y educación: percepción sobre equidad de género en relaciones y prácticas del ambiente familiar escolar. En: Educación, políticas y experiencias para Transversalizar la perspectiva de género. Comps. Castillo, C., Varela, M., Galván, L., et al. México: ITSON, pág. 52-74.

Ochoa, L. M. (2005). Investigación del currículo oculto en la educación superior: alternativa para superar el sexismo en la escuela. La Ventana, No. 21, pp. 187-227.
Ordorika, I. (2015). Equidad de género en la Educación Superior. Revista de la Educación Superior 44(174), 7-17.

Sagot M. (2006). La paz comienza en casa: la luchas de las mujeres contra la violencia y la acción estatal en Costa Rica. En Lebon, N. \& Maire, E. (coordinadoras): De lo privado a lo público: 30 años de lucha ciudadana de las mujeres en América Latina. México: Siglo XXI; UNIFEM; LASA.

Tuñón, Esperanza (coord.) (2002), Género y medio ambiente, El Colegio de la Frontera Sur / Semarnat/ Plaza y Valdés Editores, México.

UNESCO (1998). Conferencia mundial sobre la educación superior. Recuperado de: http://www.unesco.org/education/educprog/wch e/declaration_spa.htm 